Dannemann, Sarah; Neugebauer, Tjark; Schomaker, Claudia; Werning, Rolf Die LeibnizLernlandschaft. Diversität und Digitalisierung (L2D2) gestalten. Konzeptionelle Gedanken für eine inklusive Hochschullernwerkstatt an der

\title{
Leibniz Universität Hannover
}

Kramer, Kathrin [Hrsg.]; Rumpf, Dietlinde [Hrsg.]; Schöps, Miriam [Hrsg.]; Winter, Stephanie [Hrsg.]: Hochschullernwerkstätten - Elemente von Hochschulentwicklung? Ein Rückblick auf 15 Jahre Hochschullernwerkstatt in Halle und andernorts. Bad Heilbrunn : Verlag Julius Klinkhardt 2020, S. 226-237. - (Lernen und Studieren in Lernwerkstätten)

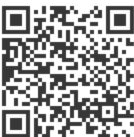

Quellenangabe/ Reference:

Dannemann, Sarah; Neugebauer, Tjark; Schomaker, Claudia; Werning, Rolf: Die LeibnizLernlandschaft. Diversität und Digitalisierung (L2D2) gestalten. Konzeptionelle Gedanken für eine inklusive Hochschullernwerkstatt an der Leibniz Universität Hannover - In: Kramer, Kathrin [Hrsg.]; Rumpf, Dietlinde [Hrsg.]; Schöps, Miriam [Hrsg.]; Winter, Stephanie [Hrsg.]: Hochschullernwerkstätten Elemente von Hochschulentwicklung? Ein Rückblick auf 15 Jahre Hochschullernwerkstatt in Halle und andernorts. Bad Heilbrunn : Verlag Julius Klinkhardt 2020, S. 226-237 - URN: urn:nbn:de:0111-pedocs-212169 - DOI: 10.25656/01:21216

https://nbn-resolving.org/urn:nbn:de:0111-pedocs-212169 https://doi.org/10.25656/01:21216

in Kooperation mit / in cooperation with:

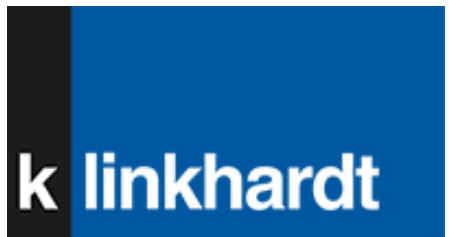

http://www.klinkhardt.de Nutzungsbedingungen

Dieses Dokument steht unter folgender Creative Commons-Lizenz: http://creativecommons.org/licenses/by-nc-sa/4.0/deed.de - Sie dürfen das Werk bzw. den Inhalt unter folgenden Bedingungen vervielfältigen, verbreiten und öffentlich zugänglich machen sowie Abwandlungen und Bearbeitungen des Werkes bzw. Inhaltes anfertigen: Sie müssen den Namen des des Werkes bzW. Inhaltes anfertigen: Sie mússen den Namen des Autors/Rechteinhabers in der von ihm Werk bzw. der Inhalt dar nicht fur kommerzielle zwecke verwendet werden. Die neu entstandenen Werke bzw. Inhalte dürfen nur unter Verwendung von Lizenzbedingungen weitergegeben werden, die mit denen dieses Lizenzvertrages identisch oder vergleichbar sind.

Mit der Verwendung dieses Dokuments erkennen Sie die Nutzungsbedingungen an.

\section{Terms of use}

This document is published under following Creative Commons-License: http://creativecommons.org/licenses/by-nc-sa/4.0/deed.en - You may copy, distribute and transmit, adapt or exhibit the work in the public and alter, transform or change this work as long as you attribute the work in the manner specified by the author or licensor. You are not allowed to make commercial use of the work. If you alter, transform, or change this work in any way, you may distribute the resulting work only under this or a comparable license.

By using this particular document, you accept the above-stated conditions of

\section{Kontakt / Contact:}

peDOCS

DIPF | Leibniz-Institut für Bildungsforschung und Bildungsinformation Informationszentrum (IZ) Bildung

E-Mail: pedocs@dipf.de

Internet: www.pedocs.de

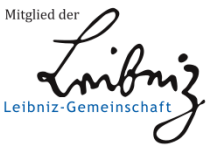




\section{Lernen und Studieren in Lernwerkstätten}

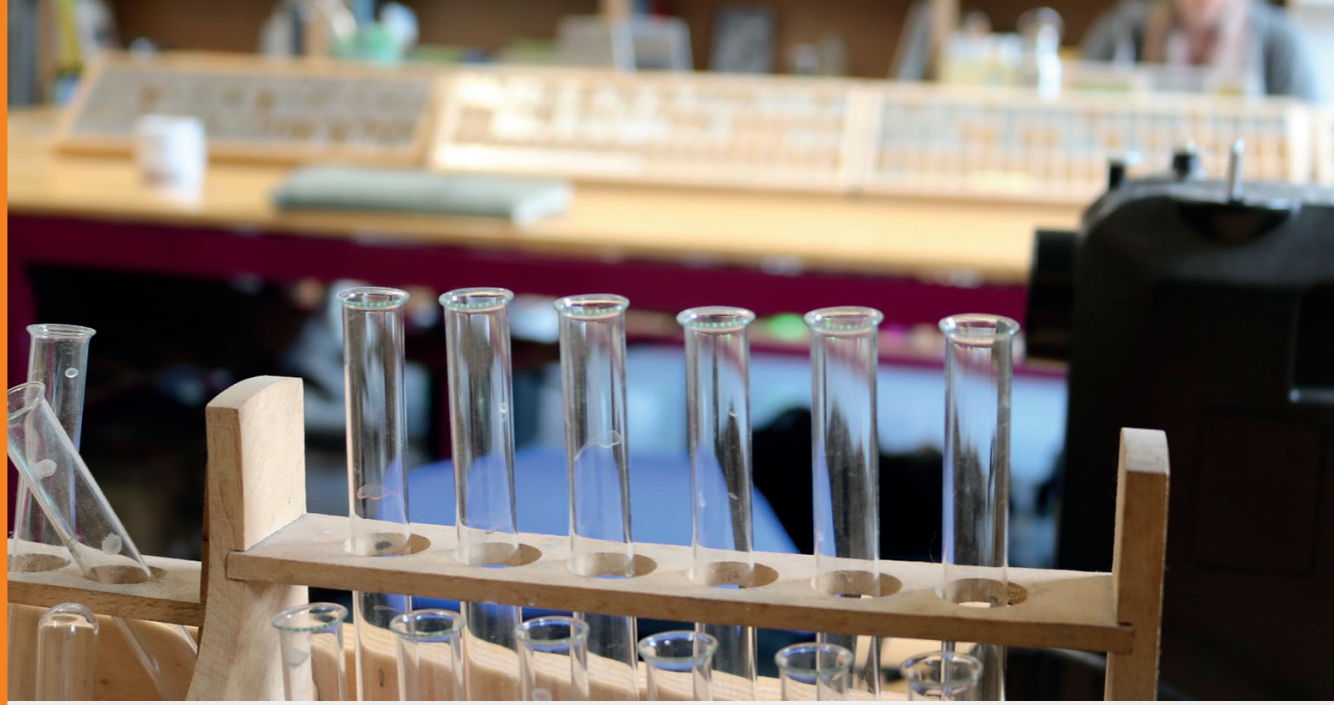

Kathrin Kramer / Dietlinde Rumpf / Miriam Schöps / Stephanie Winter (Hrsg.)

Hochschullernwerkstätten - Elemente von Hochschulentwicklung?

Ein Rückblick auf 15 Jahre Hochschullernwerkstatt in Halle und andernorts 


\section{Kramer / Rumpf / Schöps / Winter Hochschullernwerkstätten - Elemente von Hochschulentwicklung?}




\section{Lernen und Studieren in Lernwerkstätten}

Impulse für Theorie und Praxis

Herausgegeben von

Eva-Kristina Franz, Johannes Gunzenreiner, Barbara Müller-Naendrup, Hartmut Wedekind und Markus Peschel 


\section{Kathrin Kramer \\ Dietlinde Rumpf \\ Miriam Schöps \\ Stephanie Winter \\ (Hrsg.)}

\section{Hochschullernwerkstätten - Elemente von Hochschulentwicklung?}

Ein Rückblick auf 15 Jahre Hochschullernwerkstatt in Halle und andernorts 
Der vorliegende Band ist anlässlich einer Tagung des 15jährigen Bestehens der Hochschullernwerkstatt Erziehungswissenschaften in Halle im November 2020 entstanden.

Gefördert wurden die Tagung und der Band vom Zentrum für Lehrer *innenbildung, der Universitäts- und Landesbibliothek Sachsen-Anhalt sowie der Martin-Luther-Universität Halle-Wittenberg.

Dieser Titel wurde in das Programm des Verlages mittels eines Peer-Review-Verfahrens aufgenommen. Für weitere Informationen siehe www.klinkhardt.de.

Bibliografische Information der Deutschen Nationalbibliothek Die Deutsche Nationalbibliothek verzeichnet diese Publikation in der Deutschen Nationalbibliografie; detaillierte bibliografische Daten sind im Internet abrufbar über http://dnb.d-nb.de.

2020.k. (C) by Julius Klinkhardt.

Coverfoto: (C) Florian Johnke-Liese / Hochschullernwerkstatt.

Druck und Bindung: AZ Druck und Datentechnik, Kempten.

Printed in Germany 2020.

Gedruckt auf chlorfrei gebleichtem alterungsbeständigem Papier.

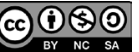

Die Publikation (mit Ausnahme aller Fotos, Grafiken und Abbildungen) ist veröffentlicht unter der Creative Commons-Lizenz: CC BY-NC-SA 4.0 International https://creativecommons.org/licenses/by-nc-sa/4.0/ 


\section{Inhalt}

Kathrin Kramer, Dietlinde Rumpf, Miriam Schöps und Stephanie Winter Einführung in den Band

\section{Teil 1: Historische Entwicklung}

Miriam Schöps und Dietlinde Rumpf

Universität mit Lernwerkstatt in Entwicklung -

Von der Materialausleihe zur Hochschuldidaktik

Hartmut Wenzel

Lernwerkstätten auch für die Sekundarstufen -

Zur Entwicklung der Hochschullernwerkstatt in Halle

Ulrike Stadler-Altmann, Susanne Schumacher, Enrico A. Emili,

Gerda Winkler und Elisabeth Dalla Torre

Hochschullernwerkstätten als Spielball der Bildungspolitik?

Die EduSpace Lernwerkstatt in der Südtiroler Lehrer*innenbildung zwischen nationalen und regionalen Bildungsinteressen

Hartmut Wedekind und Corinna Schmude

Von der Idee zum strukturell verankerten hochschuldidaktischen Prinzip -

Lernwerkstätten und Lernwerkstattarbeit im Studiengang „Erziehung und Bildung in der Kindheit" an der Alice Salomon Hochschule Berlin 68

\section{Teil 2: Wahrnehmung, Selbstverständnis, Einflüsse}

Dietlinde Rumpf und Corinna Schmude

NeHle - Internationales Netzwerk der Hochschullernwerkstätten -

Entwicklungsphasen einer Interessenvertretung und

eines gemeinsamen Begriffsverständnisses

Constantin Beyer und Florian Johnke-Liese

Hochschullernwerkstatt goes digital!

Alexandra und Michael Ritter

Drucken wie ,von gestern' in der Schule der Zukunft -

Die Schuldruckerei als Erfahrungsraum für Kinder und Studierende 
Livia Enders (geb. Makrinus) und Stephanie Winter

Studierende und Dozierende im Spannungsfeld zwischen

Theorie und Praxis - Die Hochschullernwerkstatt als Forschungsraum

John Marcus Sommer

Selbstwahrnehmung in der Hochschullernwerkstatt -

Wissenschaft zwischen Abstraktion und emotionalem Lernen

Lena Kliebe

Hochschullernwerkstatt als Raum und pädagogische Konzeption -

„Mein eigener Lernprozess“

Jerome Kampe

Geschichtsunterricht in der Lernwerkstatt!?

\section{Teil 3: Partizipation und Inklusion}

Melanie Schlag

Hochschullernwerkstatt schlägt Schlager

Kathrin Kramer

Nichtreformistische Reformen - Die Bedeutung von

Hochschullernwerkstätten auf dem Weg zu einer inklusiven Universität am Beispiel des Budgets für Arbeit

Johanna Ingenerf und Pascal Kurz

Menschenrechtsbasierte Bildung im internationalen Dialog -

Ein selbstorganisiertes Seminar mit Austausch

Ines Boban und Andreas Hinz

Hochschullernwerkstatt Halle als Möglichkeitsort -

Von WIRkstatt-Impulsen für inklusive Prozesse

Brigitte Kottmann und Alena Lensker (geb. Beckmann)

Die Lernwerkstatt und der Studiengang „Integrierte Sonderpädagogik“

an der Universität Bielefeld: Impulse, Synergien und Reflexionen

Sarah Dannemann, Tjark Neugebauer, Claudia Schomaker und Rolf Werning

Die LeibnizLernlandschaft: Diversität und Digitalisierung $\left(\mathrm{L}^{2} \mathrm{D}^{2}\right)$

gestalten - Konzeptionelle Gedanken für eine inklusive

Hochschullernwerkstatt an der Leibniz Universität Hannover 


\section{Teil 4: Reflexivität und Professionalisierung}

Kira Wybierek

Praxissemester in der Hochschullernwerkstatt -

Reflexion eines Lernprozesses

Eva Hoffart

„...da man lernt, eigene Gedanken und Ideen noch einmal zu vertiefen“ -

Theoretische Überlegungen und praktische Umsetzungen zum Reflektieren

von Lehramtsstudierenden

Edita Jung und Lena S. Kaiser

„Vielleicht romantisiere ich die Räume meiner Kindheit.“

Zugänge zu einer biographischen Reflexivität in

kindheitspädagogischen Hochschullernwerkstätten

Rolf-Torsten Kramer

Zum Problem der Professionalisierung im Lehramtsstudium und

zum Potenzial der Hochschullernwerkstatt

Mark Weißhaupt, Kathleen Panitz und Elke Hildebrandt

Die Inszenierung von „Theorie und Praxis“ sowie

„Neugier und Desinteresse“ bei der Professionalisierung

angehender Lehrpersonen in Hochschullernwerkstätten

Markus Peschel und Pascal Kihm

Hochschullernwerkstätten - Rollen, Rollenverständnisse und

Rollenaushandlungen

David Paulus, Patrick Gollub und Marcel Veber

Forschendes Lernen und Kasuistik: Überschneidungen und

Abgrenzungen bezogen auf Reflexivität in der

Hochschullernwerkstattarbeit

Pascal Kihm, Jenny Diener und Markus Peschel

Qualifizierungsprozesse und Qualifikationsarbeiten in

Hochschullernwerkstätten - Forschende Entwicklung einer

innovativen Didaktik 
10| Inhaltsverzeichnis

\section{Teil 5: Kooperation}

Georg Breidenstein, Sara Burkhardt, Thorid Rabe und Miriam Schöps

Zur Materialität des Lernens -

Anregungen aus einem interdisziplinären Forum

in der Hochschullernwerkstatt

Miriam Asmus, Kati Friebe, Mirjam Lewin und Kati Misselwitz

Entdeckendes Lernen und Digitale Medien - Ein Traumpaar .

Siglinde Spuller

Kooperation und Kooperatives Lernen als Prinzip

Hochschullernwerkstätten-adäquaten Lernens?

Eine konzeptionelle Verortung

Linda Balzer

Der Effekt selbstregulierenden und forschenden Lernens auf kooperative und individuelle Lernprozesse in der

Lernwerkstatt Religion Plural (LeRP)

Martin Lindner

Werkstattarbeit in der Biologiedidaktik -

Umstellung eines Praktikums auf Projektarbeit

Autor*innenbeschreibungen 
Sarah Dannemann, Tjark Neugebauer, Claudia Schomaker und Rolf Werning

\section{Die LeibnizLernlandschaft: Diversität und Digitalisierung $\left(L^{2} D^{2}\right)$ gestalten - Konzeptionelle Gedanken für eine inklusive Hochschullernwerkstatt an der Leibniz Universität Hannover}

\section{Zusammenfassung}

An der Leibniz Universität Hannover entsteht im Rahmen der zweiten Förderphase der Qualitätsoffensive Lehrerbildung die LeibnizLernlandschaft: Diversität und Digitalisierung $\left(L^{2} D^{2}\right)$ mit digitalen und analogen Räumen, in der Schule und Hochschule (gemeinsame) Lern- und Bildungsprozesse gestalten können. Den Kern des Konzepts bildet eine Hochschullernwerkstatt für eine partizipative und kooperative Lehrer*innenbildung. Hierdurch eröffnen sich Lern- und Entwicklungspotenziale auf unterschiedlichen Ebenen: Impulse für die Hochschuldidaktik über das Erproben oder Erleben innovativer (inter-)disziplinär durchgeführter Seminare, Impulse für eine inklusive Lehrer*innenbildung durch die entstehenden vielfältigen Forschungsanlässe, die ihrerseits die disziplinären Grenzen überschreiten können, sowie Stimuli für inklusive Schulentwicklungsprozesse an Einzelschulen bzw. übergreifende Entwicklungsarbeit.

\section{Teilhabe und Kooperation als Gestaltungsprinzipien einer inklusiven Hochschullernwerkstatt}

„Es [Arbeit in der Lernwerkstatt, Anm. der Verf.] hat mir aufjeden Fall gezeigt, dass es neue oder andere Wege gibt zu unterrichten oder zu präsentieren, dass man da auch noch mehr mit Spaß verknüpft durch diese Spiele oder durch dieses Erfinden und Entdecken. ""

1 Das Zitat stammt aus dem studentischen Forschungsprojekt von Larissa Arps, Jonas Berkhahn, Sabine Littau, Maximilian Schuster, Leon Spickschen und Fabian Steves über Anforderungen, Vor- 
In Folge der Ratifizierung der UN-Behindertenrechtskonvention (BGBL 2008, 1436f.) im Jahr 2009 verändern sich nicht nur die Organisations- und Gestaltungsprinzipien von Schulen, sondern die Bildungslandschaft befindet sich insgesamt in einem tiefgreifenden Umbruch. Deshalb ist auch die universitäre Lehrer*innenbildung aufgefordert neue Konzeptionen zu erproben, die Studierende der verschiedenen Lehramtsstudiengänge auf diese Veränderungen vorbereiten. An der Leibniz Universität Hannover entsteht im Rahmen der zweiten Förderphase der Qualitätsoffensive Lehrerbildung eine inklusive Lernlandschaft mit digitalen und analogen Räumen, in der Schulen und Universität (gemeinsame) Lern- und Bildungsprozesse gestalten können. Den Kern bildet eine Hochschullernwerkstatt als Ort für die Entwicklung und Umsetzung von Konzepten einer inklusiven Lehrer*innenbildung. An der Leibniz Universität Hannover wird bei der Ausschärfung des standortspezifischen Profils versucht, an die Tradition der Hochschullernwerkstätten anzuknüpfen. Als verbindendes Element der Hochschullernwerkstätten gilt das Potenzial „handelnder Auseinandersetzung mit einem Thema und der bewussten Reflexion des Erfahrenen auf der Grundlage einer theoriegeleiteten und zugleich theoriebegleitenden Praxis" (Schmude \& WedeKInd 2016, 11), dem sich dieses Konzept verpflichtet sieht.

Zur Gestaltung der hannoverschen Hochschullernwerkstatt werden zwei aktuelle Schwerpunkte der Lehrer*innenbildung und der Schulentwicklung, Diversität und Digitalisierung, zusammengebracht, um virtuelle und analoge Räume - im Sinne einer Lernlandschaft - zu schaffen, die von den verschiedenen, an Bildungsprozessen beteiligten, Personen multifunktional genutzt und gestaltet werden können. Die LeibnizLernlandschaft: Diversität und Digitalisierung $\left(L^{2} D^{2}\right)$ entsteht über partizipativ und kooperativ gestaltete und zu gestaltende Erfahrungs- und Lernräume, die den Rahmen für einen übergreifenden Austausch über Lernprozesse bieten. Hierdurch soll die Heterogenität der Studierenden, Dozierenden, Schüler*innen, Lehrer*innen sowie der Fakultäten, Institute, Schulformen und Schulen und ihrer theoretischen und/oder praktischen Bezüge nicht nur anerkannt werden, sondern ein grundsätzliches Gestaltungprinzip der Lernlandschaft bilden. Durch die organisatorische Verankerung an der Leibniz School of Education, die eine Querstruktur zu allen an der Lehrer*innenbildung beteiligten Fakultäten darstellt, kann die Lernlandschaft als universitäres Gemeinschaftsprojekt etabliert werden. Das Ziel ist es, trotz der Herausforderung, die individuellen Bedürfnisse der unterschiedlichen Studiengänge Lehramt an Gymnasien, Lehramt für Sonderpädagogik und Lehramt für berufsbildende Schulen zu berücksichtigen, die Teilhabeoptionen aller Akteur*innen stets zu maximieren. Dabei sind die unterschiedlichen Wünsche und Bedarfe der beteiligten Personengruppen zu

stellungen und Wünsche der Studierenden im Lehramt Sonderpädagogik zur Gestaltung von Lernwerkstätten an der Leibniz Universität Hannover. 
berücksichtigen. Strukturell wird die Partizipation durch ein Steuerungsgremium initiiert, dem Mitglieder aller Statusgruppen gleichberechtigt angehören.

Darüber hinaus sollen die virtuellen und analogen Lerngelegenheiten der Lernlandschaft Teilhabeoptionen für alle Mitglieder der Gesellschaft im Sinne einer sozialen Inklusion (JANTZEN 2018) bieten. Alle Angebote der Lernlandschaft richten sich je nach Zielsetzung an einen heterogenen Personenkreis. Im Vorfeld der Einrichtung ist deshalb zu reflektieren, welche Faktoren in der Lernlandschaft zu berücksichtigen sind, damit sie in Wechselwirkung mit den spezifischen Merkmalen von Personen und Personengruppen nicht zu Exklusion führen. Dies orientiert sich am Leitgedanken der „International Classification of Functioning“ (ICF) der WHO (DIMDI 2005), wonach bspw. die Differenzlinie Behinderung als Wechselwirkung zwischen inkorporierten Faktoren des Individuums und sozialen und materialen Umweltfaktoren verstanden wird. Über die konsequente Berücksichtigung bei der Arbeit in der Lernlandschaft kann für die Entstehungsprozesse von beispielsweise Behinderung und damit für Exklusionsprozesse sowie Einschränkungen, aber auch für Chancen der sozialen Teilhabe sensibilisiert werden. Die hannoversche Lernlandschaft bildet damit einen Ort, an dem soziale Teilhabe und Kooperation die zentralen Gestaltungselemente bilden. So werden seit mehreren Jahren in zwei Projekten des Instituts für Sonderpädagogik gemeinsame Seminare von Studierenden und Teilnehmenden ohne Hochschulzugangsberechtigung mit psychischen, geistigen und/oder körperlichen Behinderungen zu Themen historischer und politischer Bildung durchgeführt.

Infolgedessen sind Gestaltungsmöglichkeiten für vielfältige Lernsituationen und -umgebungen erforderlich, die sehr unterschiedliche Anforderungen an die räumliche und mediale Ausstattung stellen. Im aktuellen Konzept bildet ein multifunktioneller Arbeitsraum das Zentrum, der auch für klassische Arrangements genutzt werden kann. Daneben sind Arbeitsräume für Kleingruppen, eine Bühne, Freiflächen für individualisiertes Arbeiten sowie ein Raum für virtuelle Arbeitsmöglichkeiten wesentlich. Zudem ermöglichen mobile, u. a. digitale, Elemente Erfahrungs- und Lernprozesse überall auf dem Campus sowie dezentral in Schulen oder anderen Bildungseinrichtungen.

Damit wird eine Lernlandschaft geschaffen, die sowohl die Beteiligten schulischer und universitärer Bildungsprozesse als auch die drei Phasen der Lehrer*innenbildung miteinander verbinden und wechselseitige Transferpotenziale eröffnen kann. Diese Lern- und Entwicklungspotenziale eröffnen sich auf unterschiedlichen Ebenen: Zum ersten bezogen auf verschiedene organisatorischstrukturelle und organisatorisch-personelle Bedingungen, wie bspw. (inter-)disziplinär durchgeführte Seminare (vgl. Kapitel 3.1 und 3.2). In diesem Sinne kann die Lernwerkstatt zu einem „Impulsgeber einer innovationsorientierten Hochschuldidaktik“ (MülLER-NaEndrup 1997, 142) werden. Zweitens bieten diese Lernprozesse vielfältige Forschungsanlässe, die ihrerseits die disziplinären Grenzen 
überschreiten und so besondere Impulse für eine inklusive Lehrer*innenbildung geben können. Zum Dritten können durch den interdisziplinären Austausch und die erweiterten Erfahrungs- und Reflexionsmöglichkeiten über den universitären Kontext hinaus Schulentwicklungsprozesse unterstützt und Innovationen angestoßen werden. Diese können sich sowohl auf die schulische Inklusion an Einzelschulen beziehen als auch inklusive Schulentwicklung durch universitäre Lern- und Bildungsprozesse in Zusammenarbeit mit Schulen und Studienseminaren ermöglichen.

Im Folgenden werden theoretisch-konzeptionelle Grundlagen (Kap. 2) der Lernlandschaft und ihre praktische Umsetzung (Kap. 3) exemplarisch ausgeführt.

\section{Potenziale der LeibnizLernlandschaft: Diversität und Digitalisierung}

\subsection{Reflexive Handlungsfähigkeit}

Die erweiterte Heterogenität der Lernenden und veränderte Unterrichtsrealitäten erfordern veränderte Lerngelegenheiten in der Lehrer*innenbildung. Neben einer wertschätzenden und anerkennenden Haltung gegenüber der Heterogenität (TERHART 2015) sind Wissen und Handlungsfähigkeiten, bspw. über diagnostische Verfahren und differenzierende Lernangebote sowie Prüfungsformen wesentlich, die eine individuelle Förderung erlauben. Zudem sind Fähigkeiten zur Kooperation mit Kolleg*innen auch anderer Disziplinen relevant (Werning 2018), um über die unterschiedlichen Expertisen verschiedene Ressourcen der Schüler*innen ansprechen zu können. Um ein begründetes und theoriegeleitetes Handeln in den hochkomplexen, stets von Ungewissheit geprägten Unterrichtssituationen zu ermöglichen sowie das eigene Unterrichtshandeln und das Handeln anderer, bspw. im Rahmen interdisziplinärer Zusammenarbeit, diskutieren und verändern zu können, sind zudem Reflexionsfähigkeiten erforderlich (HeLsPER 2016). In Universitäten können Räume entstehen, die Studierenden und Dozierenden aber auch Referendar*innen und Lehrpersonen - Möglichkeiten für einen biografisch-selbstreflexiven Umgang mit dem Ungewissen bieten. Dies wird als zentral für die Professionalisierung der Akteur*innen angesehen (Amrhein 2017, 125). Im aktuellen Diskurs wird betont, dass sich Professionalität in diesem Bereich durch Multiperspektivität (Helsper 2016) oder Meta-Reflexivität (CRAMER \& Drahmann 2019) auszeichnet: Lehrende sind dazu in der Lage, eine Situation aus verschiedenen Perspektiven, bspw. bezogen auf fachwissenschaftliche, fachdidaktiktische und erziehungswissenschaftliche Theorien zu betrachten und die Grundlagen dieser Theorien aufeinander zu beziehen. 
Im LeibnizPrinzip wurde das Leitbild der Reflexiven Handlungsfähigkeit für die Lehrer*innenbildung an der Leibniz Universität entwickelt (GILLEN 2015, DANNEMANN ET AL. 2019). Betont wird - neben den fachlichen, fachdidaktischen und erziehungswissenschaftlichen Kompetenzen - die Bedeutung von Reflexionen, die es ermöglichen, mit der steten Ungewissheit in Unterrichtssituationen umzugehen und die Strukturen und Bedingungen von Unterricht aus verschiedenen Perspektiven betrachten zu können (Helsper 2016). Im Kern dieses Leitbilds stehen eine praxisbezogene Theoriebildung, eine kooperative Professionalisierung und Reflexivität. Letztere umfasst neben der Verbesserung eigener Handlungsweisen und der Erweiterung von Handlungsoptionen auch das In-Frage-Stellen eigener (erkenntnis-)theoretischer Annahmen (MüLLER 20IO). Eine multifunktionale Lernwerkstatt, die unterschiedlichste Lernwege eröffnet, kann bereits in der universitären Lehrer*innenbildung Raum für irritierende Erfahrungen bieten - und Lernräume, in denen neue Möglichkeiten erlebt, erprobt und wertgeschätzt werden können (vgl. 3.1). Indem die perspektivische Vielfalt bereits in der ersten Phase der Lehrer*innenbildung auch praktisch erlebt werden kann, besteht die Möglichkeit, Theorie und Praxis so aufeinander zu beziehen, dass sowohl deren singuläre Potenziale als auch der Wert von Synthesewirkungen beider Bezugssysteme für das spätere berufliche Handeln erkannt werden können (vgl. 3.2). Lernwerkstätten können hier Erfahrungs- und Lernräume für strukturell, personell und situationell orientierte Irritationen darstellen, in denen eigene Routinen reflektiert und Veränderungen von Handlungsweisen möglich sind.

\subsection{Schulentwicklung}

Die Bedeutung der Einzelschule als pädagogische Handlungseinheit zur Verbesserung der Qualität von Bildungs- und Erziehungsprozessen (vgl. z. B. FEND 2008) gewinnt im Zuge der inklusiven Schulentwicklung weiter an Bedeutung. Inklusive Schulentwicklung öffnet eine auf interne Prozesse gerichtete Perspektive, erweitert diese um Schule-Umwelt-Bedingungen und betont, dass Bildungs- und Lernprozesse von Kindern und Jugendlichen nicht ausschließlich in Schulen, sondern an verschiedenen Lernorten stattfinden (ERHORN \& SCHWier 2016). Aufgrund der Erweiterung der Wirkbeziehung und durch die Anforderungen der flächendeckenden Implementierung inklusiver Schulkulturen ist es notwendig, pädagogische Konzepte zu entwickeln, „die einen allgemeinen Erziehungsauftrag mit den speziellen Bedürfnissen benachteiligter Personengruppen verbinden" (WERning \& Neugebauer im Druck). Damit kann Inklusion in diesem Zusammenhang sowohl als Stimulus für Entwicklungsprozesse als auch als Dauerauftrag beschrieben werden (Werning \& ARndt 2019). Über eine phasenübergreifende Zusammenarbeit im Rahmen der Lernwerkstattsarbeit sollen Studierende und Lehrkräfte für diese Prozesse sensibilisiert werden, da inklusive Schul- und Unterrichtsentwicklung eine disziplinübergreifende Zusammenarbeit von Pädogog*innen erfordert, 
die „über entsprechende inklusionsbezogene Kompetenzen, epistemologische Überzeugungen und berufsbezogene Werthaltungen verfügen“ (LüTJE-KLOSE \& Neumann 2018, 129).

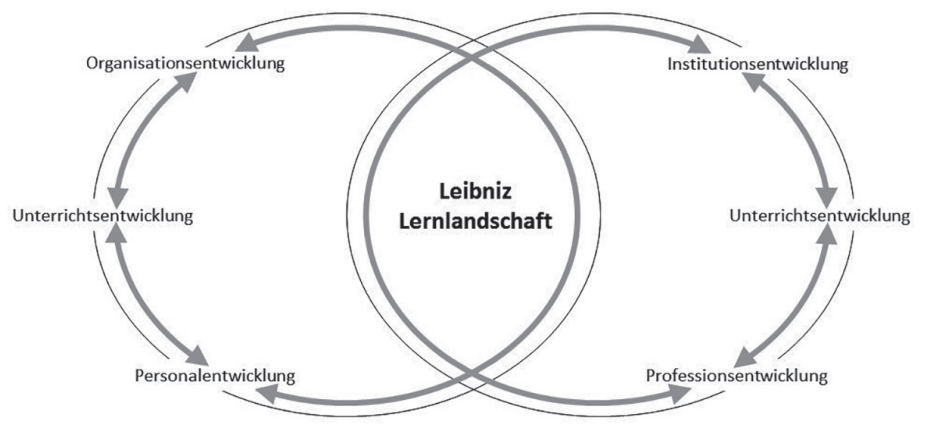

Abb. 1: Symbiosepotenziale der LeibnizLernlandschaft für schulisch und universitär angestoßene (Schul-)Entwicklungsprozesse; eigene Darstellung

Im Kontext der LeibnizLernlandschaft sollen Studierende und Lehrkräfte die Chance erhalten, gemeinsam Schulentwicklungsarbeit zu betreiben. Der individuelle, wertschätzende Umgang mit Diversität an der Einzelschule oder die Nutzung digitaler Medien zur Unterrichtsgestaltung können Anlässe für Entwicklungsprozesse sein. Ausgehend von der klassischen Trias der Schulentwicklung nach RoLfF (2016) aus Organisations-, Unterrichts- und Personalentwicklung kann die Lernlandschaft als integrativer Ort diesen Prozess aktiv unterstützen. Dabei werden Einzelschulen als Organisationen verstanden. Im Allgemeinen können Universitäten nur indirekt Schulentwicklung - bezogen auf die Lehr-Lernprozesse im Unterricht, das Personalmanagement und die Strukturen der Schulverwaltung und -organisation - von Einzelschulen mitgestalten. Daher können im Rahmen der LernLandschaft stattfindende allgemeine Schulentwicklungsprozesse in Anlehnung an die klassische Trias als Institutions-, Unterrichts- und Professionsentwicklung beschrieben werden (Abb.1). Über die gemeinsame Arbeit von Universität und Schule in der Lernlandschaft können beide Schulentwicklungsansätze miteinander verbunden werden. Für die Studierenden ergibt sich die Chance, bereits im Studium aktiv an Schulentwicklungsprozessen mitzuarbeiten und durch diese theoretische und praktische Arbeit ihre individuellen professionellen Innovationskompetenzen zu erweitern im Sinne der KMK-Bildungsstandards für die Lehrer*innenbildung (KMK 2019). Für Lehrkräfte ergibt sich die Chance, eine inklusive Schul- und Unterrichtskultur unter Berücksichtigung der spezifischen Anforderungen der Einzelschule im Diskurs mit der Universität als Partner im Entwicklungsprozess mitzugestalten. Für beide Gruppen kann die Lernlandschaft 
einen geschützten Raum bieten, der unterstützen und gleichzeitig praktische Erfahrungen eröffnen kann. Dem hier beschriebenen Modell liegt die Vorstellung eines Review-Verfahrens im Sinne des Qualitätsmanagements zu Grunde. Verfahren des Peer Review dienen dem Austausch unter Kolleg*innen verschiedener Schulen zur Weiterentwicklung durch kollegiales Feedback (z. B. Gieske-Roland ET AL. 2014). Da der Austausch in der Lernlandschaft zwischen Novizen und erfahrenen Lehrkräften erfolgt, betont die Bezeichnung als Peers hier den erwünschten Austausch in gegenseitiger Wertschätzung. In dieser Begegnung werden zusätzliche Chancen für Irritation und Innovation gesehen.

\section{Praktische Einblicke}

\section{1 ,Frei-Räume' denken und planen - Inklusive Sachbildungsprozesse im Kontext von Lernwerkstätten initiieren, unterstützen, implementieren}

Angelehnt an das Bildungsverständnis von Wolfgang KLAFKI (1992) ist es Ziel des Faches Sachunterricht, „Schülerinnen und Schüler darin zu unterstützen, ihre natürliche, kulturelle, soziale und technische Umwelt sachbezogen zu verstehen, sie sich auf dieser Grundlage bildungswirksam zu erschließen und sich darin zu orientieren, mitzuwirken und zu handeln" (GDSU 2013, 9). Insbesondere mit Blick auf den Qualifikationsschwerpunkt Überzeugungen und Haltungen im Kontext einer inklusions-/diversitätssensiblen Lehrer*innenbildung zeigen aktuelle Forschungsergebnisse nachdrücklich auf (vgl. u. a. Junge 2019), dass die Reflexion von Haltungen und Überzeugungen immanenter Bestandteil von Lehrer*innenbildung sein muss, um das Wirksamwerden von Deutungsmustern in Praktiken des zukünftigen Tätigkeitsfeldes reflektieren zu können. Zugleich wird im Kontext der Forschungen zu diesem Professionalisierungsaspekt inklusionssensibler Lehrer*innenbildung aber auch deutlich, dass gängige Lehr-Lernformate der Hochschulbildung diesem Anspruch nicht gerecht werden (vgl. ebd., Oldenburg 2019).

Die Idee zum ,Frei-Raum' lehnt sich an die grundlegende Idee der Kunst- und Wunderkammern der Renaissance und deren moderne Adaptionen an (vgl. Mauriès 2011, Spenlé 2015), wie sie z. B. in der restaurierten Kunst- und Naturalienkammer der Franckeschen Stiftungen in Halle sichtbar ist (vgl. u. a. MülLER-BAHLKe 1998). Wunderkammern verstehen sich als wissenschaftliche Sammlungen aller Art, in denen mehr oder weniger systematisch Gegenstände nach ihrer Herkunft oder thematisch geordnet in den Mittelpunkt der Betrachtung gestellt werden (vgl. Becher \& Schomaker 2018). Lernprozesse in diesem Frei-Raum verlaufen entlang der eigenen Interessen und Fähigkeiten der Lernen- 
den, sich mit den vielfältigen Phänomenen ihrer Lebenswelt auseinanderzusetzen, um begründet Position beziehen, hinter die Dinge blicken und an gesellschaftlichen Debatten partizipieren zu können. Darin spiegelt sich auch der Anspruch des Faches Sachunterricht wider: Ausgehend von Dingen, Gegenständen, dem physisch Sachlichen werden eigene Fragen entwickelt, die mit je spezifischer Fokussierung in eine wissenschaftsbezogene Sachauseinandersetzung führen (können).

Mit Blick auf diese Zielsetzungen haben sich Studierende der Sonderpädagogik mit dem Unterrichtsfach Sachunterricht verschiedene Ansätze zur Auseinandersetzung mit Dingen bzw. den Zielsetzungen derartiger Lern- und Bildungsprozesse (u. a. Elschenbroich 2010, Kämpf-JANSEn 2000, Duncker \& Lieber 2013, Duncker 2018) erarbeitet und auf die Auseinandersetzung mit Dingen und ihren damit verbundenen individuellen Fragen bezogen. Es entstanden verschiedene Entwürfe für die Gestaltung von Räumen, in denen eine derartige Auseinandersetzung angeregt wird. Diese verschiedenen Entwürfe erprobten die Studierenden mit Schüler*innen, um die Möglichkeiten und Grenzen der Raumideen in Bezug auf die Zielsetzungen einer Auseinandersetzung mit Dingen im Kontext sachunterrichtsdidaktischer Überlegungen zu reflektieren. In einem nächsten Schritt erarbeiteten sich Studierende weiterer Gruppen Grundlagen didaktischer Zugänge (u. a. Duncker 2018, Kämpf-Jansen 2000, Alexander 1995) und entwickelten mit Kenntnis der verschiedenen Raumideen konkrete didaktische Handlungskonzepte für die Begegnung, Erforschung und Auseinandersetzung mit Dingen in dem so geschaffenen Lern- und Bildungsraum. Die Arbeit mit den Studierenden und ihre Rückmeldungen zu diesen Seminarangeboten zeigen, dass für manche ein derartiger Zugang zu Fragestellungen ungewohnt, irritierend ist, von anderen jedoch schnell als Ort im o.g. Sinne verstanden und angenommen wird.

Mit der Einrichtung eines ,Frei-Raums' (vgl. Strutz 2019) an der Leibniz Universität soll es Studierenden von Beginn an in ihrem Studium ermöglicht werden, individuelle und gemeinschaftliche Sachbildungsprozesse zu erfahren und in diesem Kontext individuelle Deutungsmuster zu reflektieren.

\subsection{Unterricht anders denken - Seminarformate in interdisziplinären Tan- dems als Möglichkeit zur Professionalisierung ${ }^{2}$}

Traditionell erfolgt die Lehrer*innenbildung fach- und schulformbezogen separiert, gemeinsames Lehren und Lernen findet nur selten statt. In der Lernlandschaft können die Bedeutung der Perspektiven anderer Disziplinen und von Kooperation erfahrbar werden. Ausgehend von der Wichtigkeit der interdisziplinären Kooperation für eine inklusive Schulentwicklung zielt das hier vorgestellte

2 Die Entwicklung und Erprobung des Seminarformats wird im Rahmen des Hochschulpakts 2020 „Innovation plus“ (Dannemann, Nehring, Neugebauer, Werning) gefördert. 
Seminarformat darauf ab, dass sich Studierende der Sonderpädagogik (M.Ed., Förderschwerpunkt Lernen) und des Lehramts an Gymnasien (M.Ed., Unterrichtsfach Biologie) als Tandem forschend mit inklusivem Biologieunterricht auseinandersetzen. Auch die Dozierenden bilden ein Tandem aus beiden Disziplinen. Orientiert an einem Collaborative Training Programme (Stayton \& McCollum 2002) werden die theoretischen Fundierungen der beteiligten Disziplinen mit den Erfahrungen einer - im besten Fall - kooperativen Praxis verbunden. Im ersten Teil des Seminars werden fallbasiert sonderpädagogische, biologische und biologiedidaktische Grundlagen erarbeitet. Hierzu gehören der jeweilige fachliche Gegenstand, das Konstrukt der Schüler*innenvorstellungen (Gropengiesser 2006), Lernbarrieren sowie Möglichkeiten für Individualisierung und Kooperation im Unterricht an weiterführenden Schulen. Die eingesetzten Fälle umfassen Video- (Dannemann 2018) sowie Textvignetten. Bei der Erarbeitung können sich die Tandems durch ihre jeweils komplementären Expertiseprofile wechselseitig unterstützen. Im zweiten Teil des Seminars beobachten die Tandems inklusiven Unterricht an Partnerschulen und erstellen Protokolle. Im Fokus stehen Fragen zur Gestaltung der Kooperation mit Bezug zum Lernen von Schüler*innen. Durch die forschende Perspektive werden die Studierenden zum einen vom direkten Handlungsdruck entlastet. Zum anderen bietet das kritische Reflektieren von Unterricht Potenziale für berufsbezogene Professionalisierungsprozesse und kann eine forschende Haltung unterstützen. In der abschließenden Phase des Seminars werden die Unterrichtsprotokolle gemeinsam rekonstruktiv mit Blick auf inklusiven Unterricht sowie Kooperation interpretiert. Damit kann das Seminar über die interdisziplinäre Gestaltung, den Fokus auf Theorie-Praxis-Verbindungen und den - bestenfalls - kooperativen Charakter der Zusammenarbeit Beiträge zur Reflexiven Handlungsfähigkeit (vgl. 2.2) leisten. Die fallbasierten Analysen werden den Fachgruppen der Partnerschulen zur eigenen Unterrichtsentwicklung zur Verfügung gestellt.

Die Lernlandschaft bietet für die Seminare sowohl einen strukturellen als auch konzeptuellen Rahmen: Neben Räumen zur gemeinsamen Arbeit der Tandems bietet der interdisziplinäre Begegnungsort auch über den formellen Seminarkontext hinaus einen vielfältigen Erfahrungsraum mit den verschiedenen Partizipierenden am Bildungssystem. Die sowohl formellen als auch informellen Begegnungen bieten den Studierenden Möglichkeiten, Perspektiven und theoretische Ansätze anderer Disziplinen, bspw. zu Lernen und Unterricht oder der Rolle der Lehrenden und der Lernenden, kennenzulernen und zu diskutieren. Zumindest für die Studierenden des Lehramts an Gymnasien stellt das Seminar häufig die erste Begegnung mit Schüler*innen dar, für die ein sonderpädagogischer Förderbedarf festgestellt wurde. Für angehende Sonderpädagog*innen ist die Fachlichkeit des Gegenstands eine Hürde in der gemeinsamen Arbeit auf Augenhöhe, die in diesem Seminar exemplarisch er- und bearbeitet werden kann. 


\section{Diskussion}

Durch die Gestaltung einer inklusiven Lernlandschaft mit den Schwerpunkten Diversität und Digitalisierung ergeben sich Potenziale für inklusive Bildungsprozesse. Darüber, dass Studierende das eigene Lernen in unterschiedlichen Rollen (pädagogischer Doppeldecker) und damit das Spannungsfeld von Instruktion und Konstruktion erleben, können sie irritierende Erfahrungen machen, die über Reflexionsprozesse individuelle Innovationen in Haltung, Denken und Handeln anstoßen können. Auch Lehrende an Schule oder Hochschule können über eine theorie- und erfahrungsgeleitete Reflexion der eigenen Praxis „individuell an Entwicklungsaufgaben der Professionalisierung [...] arbeiten und Kompetenzen für den inklusiven Unterricht auf[...]bauen und [...] entwickeln" (vgl. Franz \& SAnsour 2016, 58). Dazu trägt auch die Verbindung aus Forschung und Lehre bei, die neben dem in-Beziehung-Setzen von Theorie und Praxis zu neuen Erkenntnissen über das Lernen in Lernwerkstätten beitragen kann. Insbesondere zur Stärkung der forschenden Haltung der beteiligten Akteur*innen können kasuistische Arbeitsformate eine Unterstützung im Sinne der Ausbildung einer reflexiven Handlungsfähigkeit bieten.

Nicht zuletzt bieten sich über den institutions- und phasenübergreifenden Austausch, an dem viele Beteiligte partizipieren, Möglichkeiten zur Vernetzung und Mitgestaltung, die zu einer Ausgestaltung einer kommunalen bzw. regionalen inklusiven Bildungslandschaft (Тномs ET AL. 2019) und ihrer steten Weiterentwicklung beitragen können.

\section{Literatur}

Alexander, Christopher (1995): Eine Mustersprache. Wien: Locker.

Amrhein, Bettina (2017): Professionalisierung für Inklusion: Akteurinnen und Akteure der Sekundarstufe zwischen Determination und Emergenz. In: Lindmeier, Christian \& Weiss, Hans (Hrsg.): Pädagogische Professionalität im Spannungsfeld von sonderpädagogischer Förderung und inklusiver Bildung. Weinheim: Beltz Juventa, 118-133.

Becher, Andrea \& Schomaker, Claudia (2018): Sammeln, aufbewahren, ordnen und präsentieren. Dinge in Ordnung bringen. Grundschule Sachunterricht H. 79, 18-28.

BGBL (Bundesgesetzblatt) (2008). Gesetz zu dem Übereinkommen der Vereinten Nationen vom 13. Dezember 2006 über die Rechte von Menschen mit Behinderungen sowie zu dem Fakultativprotokoll vom 13. Dezember 2006 zum Übereinkommen der Vereinten Nationen über die Rechte von Menschen mit Behinderungen. Vom 21. Dezember 2008. Berlin: Bundesanzeiger, 1419-1457.

Cramer, Colin \& Drahmann, Martin (2019): Professionalität als Meta-Reflexiviät. In: Syring, Marcus \& Weiss, Sabine (Hrsg.): Lehrer(in) sein - Lehrer(in) werden - die Profession professionalisieren. Heilbronn: Klinkhardt, 17-33.

Dannemann, Sarah (2018): Rethinking Lesson Planning - Using Video Vignettes as Cases in ELearning Scenarios. In: Finlayson, Odilla; McLoughlin, Eilish; Erduran, Sibel \& Childs, Peter (Hrsg.): Electronic Proceedings of the ESERA 2017 Conference. Research, Practice and Collaboration in Science Education, Part 13/208. Dublin, Ireland: Dublin City University, 1881-1891. 
Dannemann, Sarah; Gillen, Julia; Krüger, Alexandra; Oldenburg, Maren; Sterzik, Linda \& von Roux, Yvonne (2019): Zur Entwicklung des Leitbilds der Reflektierten Handlungsfähigkeit Herausforderungen und Chancen für die erste Phase der Lehrer*innenbildung. In: Dannemann, Sarah; Gillen, Julia; Krüger, Alexandra \& von Roux, Yvonne (Hrsg): Reflektierte Handlungsfähigkeit in der Lehrer*innenbildung - Leitbild, Konzepte und Projekte. Berlin: Logos Verlag, 15-36.

DIMDI (Deutsches Institut für Medizinische Dokumentation und Information) (2005): Internationale Klassifikation der Funktionsfähigkeit, Behinderung und Gesundheit. Im Internet: https:// www.dimdi.de/dynamic/.downloads/klassifikationen/icf/icfbp2005.zip.

DunCKer, Ludwig (2018): Wege zur ästhetischen Bildung. Anthropologische Grundlegung und schpädagogische Orientierungen. München: kopaed.

Duncker, Ludwig \& Lieber, Gabriele (2013): Bildliteralität und ästhetische Alphabetisierung. München: kopaed

ERHorn, Jan \& Schwier, Jürgen (Hrsg.) (2016): Pädagogik außerschulischer Lernorte. Eine interdisziplinäre Annäherung. Bielfeld: transcript.

Elschenbroich, Donata (2010): Die Dinge. Expeditionen zu den Gegenständen des täglichen Lebens. München: Kunstmann.

Franz, Eva-Kristina \& SAnsour, Teresa (2016): Alle(s) drin? - Lernwerkstattarbeit und Professionalisierung im Kontext von Inklusion. In: Schmude, Corinna \& Wedekind, Hartmut (Hrsg.): Lernwerkstätten an Hochschulen. Orte einer inklusiven Pädagogik. Bad Heilbrunn: Klinkhardt Verlag, 51-64.

FEND, Helmut (2008): Schule gestalten. Heidelberg: Springer.

GDSU (Gesellschaft für Didaktik des Sachunterrichts) (2013): Perspektivrahmen Sachunterricht. Vollständig überarbeitete und erweiterte Auflage. Bad Heilbrunn: Klinkhardt.

GDSU (Gesellschaft für Didaktik des Sachunterrichts) (2019): Qualitätsrahmen Lehrerbildung Sachunterricht und seine Didaktik. Bad Heilbrunn: Klinkhardt.

Gieske-Roland, Mario; Buhren, Claus G. \& Rolff, Hans-Günter (2014): Peer Review an Schulen. Unterrichtsentwicklung durch gegenseitige Schulbesuche. Weinheim: Beltz.

Gillen, Julia (2015): Das Leibniz-Prinzip in der Lehrerbildung. Ein Leitbild für die Vielfalt. Unimagazin, 03/04, 14-17.

Gropengießer, Harald (2006): Lebenswelten, Denkwelten, Sprechwelten - Wie man die Vorstellungen der Lerner verstehen kann. Baltmannsweiler: Schneider Hohengehren.

Helsper, Werner (2016): Lehrerprofessionalität - der strukturtheoretische Ansatz. In: Rothland, Martin (Hrsg.): Beruf Lehrer/Lehrerin. Ein Studienbuch. Münster: Waxmann, 103-125.

JantZen, Wolfgang (2018): Soziale Inklusion. In: WaLM, Maik; Häcker, Thomas,; Radisch, Falk \& KRÜGER, Anja (Hrsg.): Empirisch-pädagogische Forschung in inklusiven Zeiten. Konzeptualisierung, Professionalisierung, Systementwicklung. Bad Heilbrunn: Klinkhardt, 88-102.

Junge, Alice (2019): ,Wir sind alle wunderbar, egal wie verschieden wir Menschen sind'. Eine rekonstruktive Studie zur Entwicklung der professionellen Haltung im Kontext inklusionsorientierter Lehrer*innenbildung. Hannover, unveröffentlichte Dissertation.

KÄMPf-Jansen, Helga (2000): Ästhetische Forschung. Wege durch Alltag, Kunst und Wissenschaft zu einem innovativen Konzept ästhetischer Bildung. Köln: Salon Verlag.

KLAFKI, Wolfgang (1992): Allgemeinbildung in der Grundschule als Bildungsauftrag des Sachunterrichts. In: Lauterbach, Roland; Köhnlein, Walter; Spreckelsen, Kay \& Klewitz, Elard (Hrsg.): Brennpunkte des Sachunterrichts. Kiel: IPN und GDSU, 11-31.

KMK [Kultusministerkonferenz] (2019). Standards für die Lehrerbildung: Bildungswissenschaften. Im Internet: http://www.kmk.org/fileadmin/Dateien/veroeffentlichungen_beschluesse/2004/ 2004_ 12_16-Standards-Lehrerbildung-Bildungswissenschaften.pdf.

MAuriès, Patrick (2011): Das Kuriositätenkabinett. Köln: Dumont. 
Lütje-Klose, Birgit \& Neumann, Phillip (2018): Professionalisierung für eine inklusive Schule. In: Lütje-Klose, Birgit; Riecke-Baulecke, Thomas \& Werning, Rolf (Hrsg.): Basiswissen Lehrerbildung: Inklusion in Schule und Unterricht. Grundlagen in der Sonderpädagogik (1. Aufl.). Seelze: Klett Kallmeyer, 129-151.

MüLleR, Stefan (2010): Reflex, Reflektion und Reflexion. Dimensionen von Reflexivität in der Lehramtsausbildung. In: Liebsch, Katharina (Hrsg.): Reflexion und Intervention. Zur Theorie und Praxis schulpraktischer Studien. Baltmannsweiler: Schneider Hohengehren, 27-52.

Müller-Bahlke, Thomas J. (1998): Die Wunderkammer. Die Kunst- und Naturalienkammer der Franckeschen Stiftungen zu Halle (Saale). Halle/Saale: Verlag der Franckeschen Stiftungen.

Müller-Naendrup, Barbara (1997): Lernwerkstätten an Hochschulen. Ein Beitrag zur Reform der Primarstufenlehrerausbildung. Frankfurt am Main: Peter Lang.

Oldenburg, Maren (2019): Lernendenperspektiven - Eine Herausforderung für die inklusionorientiere Lehrer*innenbildung? In: Schomaker, Claudia \& Oldennburg, Maren (Hrsg.): Forschen, Reflektieren, Bilden. Forschendes Lernen in der diversitätssensiblen Hochschulbildung. Baltmannsweiler: Schneider, 137-147.

Rolff, Hans-Günter (2016): Schulentwicklung kompakt. Modelle, Instrumente, Perspektiven. Weinheim und Basel: Beltz.

Schmude, Corinna \& Wedekind, Hartmut (2016): Lernwerkstätten an Hochschulen. Orte einer inklusiven Pädagogik. Bad Heilbrunn: Klinkhardt.

Spenlé, Virgine (2015): Die Wunderkammer: Eine Einführung. In: me Collectors Room/Stiftung Olbricht (Hrsg.): Lehrmaterial Wunderkammerschiff. Berlin: me Collectors Room/Stiftung Olbricht, 4-6.

Stayton, Vicki D. \& McCollum, Jeanette (2002): Unifying General and Special Education: What Does the Research Tell Us? Teacher Education and Special Education H. 25, 3, 211-218.

Strutz, Hildegard (2019): Forschendes Lernen im ,Frei-Raum'. In: Schomaker, Claudia \& OldenBURG, Maren (Hrsg.): Forschen, Reflektieren, Bilden. Forschendes Lernen in der diversitätssensiblen Hochschulbildung. Baltmannsweiler: Schneider, 246-257.

Terhart, Ewald (2015): Umgang mit Heterogenität: Anforderungen an Professionalisierungsprozesse. In: Fischer, Christian (Hrsg.): (Keine) Angst vor Inklusion. Herausforderungen und Chancen gemeinsamen Lernens in der Schule. Münster, New York: Waxmann, 69-83.

Thoms, Sören; Meser, Kapriel \& Neugebauer, Tjark (2019): Inklusive Schulen in kommunalen Bildungslandschaften. In: Hartmann, Magdalena; Hummel, Myriam; Lichtblau, Michael; Löser, Jessica M. \& Thомs, Sören (Hrsg.): Facetten inklusiver Bildung. Nationale und internationale Perspektiven auf die Entwicklung inklusiver Bildung. Bad Heilbrunn: Klinkhardt, 126-134.

Werning, Rolf \& Neugebauer, Tjark (im Druck): Sonderpädagogik und Rehabilitationspädagogik in der Lehrerinnen- und Lehrerbildung. In: CRAmer, Colin; KöNIG, Johannes; Rothland, Martin \& BLÖMEKE, Sigrid (Hrsg.): Handbuch Lehrerinnen- und Lehrerbildung. Bad Heilbrunn: Klinkhardt.

Werning, Rolf \& ARndt, Ann-Kathrin (2019): Inklusive Schulentwicklung. Herausforderungen und Handlungsperspektiven für die Schulleitung. Schulleitung und Schulentwicklung H. 3, Stuttgart: Raabe Verlag.

Werning, Rolf (2018): Gemeinsam inklusiv Unterrichten. Grundlagen zur Kooperation von Lehrkräften im inklusiven Unterricht. In: Schule inklusiv H. 1, 4-8. 